\title{
Health and genetic ancestry testing: time to bridge the gap
}

\author{
Andrew Smart ${ }^{1 *}$ (D), Deborah A. Bolnick ${ }^{2}$ and Richard Tutton ${ }^{3}$
}

\begin{abstract}
Background: It is becoming increasingly difficult to keep information about genetic ancestry separate from information about health, and consumers of genetic ancestry tests are becoming more aware of the potential health risks associated with particular ancestral lineages. Because some of the proposed associations have received little attention from oversight agencies and professional genetic associations, scientific developments are currently outpacing governance regimes for consumer genetic testing.

Main text: We highlight the recent and unremarked upon emergence of biomedical studies linking markers of genetic ancestry to disease risks, and show that this body of scientific research is becoming part of public discourse connecting ancestry and health. For instance, data on genome-wide ancestry informative markers are being used to assess health risks, and we document over 100 biomedical research articles that propose associations between mitochondrial DNA and $Y$ chromosome markers of genetic ancestry and a wide variety of disease risks. Taking as an example an association between coronary heart disease and British men belonging to $Y$ chromosome haplogroup I, we show how this science was translated into mainstream and online media, and how it circulates among consumers of genetic tests for ancestry. We find wide variations in how the science is interpreted, which suggests the potential for confusion or misunderstanding.

Conclusion: We recommend that stakeholders involved in creating and using estimates of genetic ancestry reconsider their policies for communicating with each other and with the public about the health implications of ancestry information.
\end{abstract}

Keywords: Direct-to-consumer genetic tests, Genetic ancestry, Disease/Health risk, Regulation, Social implications, Public understanding

\section{Background}

While genetic ancestry tests marketed to consumers do not currently claim to provide information about disease risk, it is becoming increasingly difficult to keep information about ancestry separate from information about health. In this article, we consider the recent and unremarked upon growth in genetic tests and biomedical studies linking markers of genetic ancestry to various diseases and medical conditions. These developments are becoming part of public discourse connecting ancestry and health, but because genetic testing companies, oversight agencies, and professional genetic associations have largely treated health and ancestry genetic tests as

\footnotetext{
* Correspondence: a.smart@bathspa.ac.uk

'Department of Sociology, Bath Spa University, Newton Park, Bath BA2 9BN, UK

Full list of author information is available at the end of the article
}

independent and distinct, little guidance is available to help consumers understand and interpret the reported connections between genetic ancestry and disease risk. Consequently, when such findings circulate in the public realm, consumers learn that there may be health risks tied to their genetic ancestry even though companies do not report those associations. There is therefore potential for confusion or misunderstanding that is problematic for both consumers and the scientific community. We argue that the various stakeholders in genetic ancestry testing need to reconsider what they communicate about the health implications of ancestry information, both to the public and to each other, in order to effectively bridge the gap that currently exists in policies and consumer guidance regarding genetic tests for ancestry and health. 


\section{The gap between genetic tests for health and ancestry}

Genetic ancestry tests were first marketed directly to consumers in 2000, for the purpose of reconstructing genealogies and investigating personal genetic heritage. They quickly became the most popular of all consumer genetic testing services, and more than three million individuals have reportedly purchased these tests to date $[1,2]$. Over the last 15 years, companies [3], regulators [4] and professional scientific associations [5, 6] have treated ancestry genetic tests differently than medical or health-oriented genetic tests. Tests that make healthrelated claims or have implications for the prevention, diagnosis, or treatment of disease have been subject to greater scrutiny and oversight, as regulators have sought to ensure that potentially life-changing healthcare decisions are not made on the basis of poor quality information or with a lack of appropriate medical knowledge, advice, and support [7]. Genetic ancestry tests have received less attention from legislators, policy makers and regulatory agencies because they are not marketed explicitly for disease diagnosis, treatment, or prevention, and have thus been seen as more "recreational", less consequential, and less ethically problematic. This differentiation between ancestry and health genetic testing has seemed appropriate because the two types of tests have had such different applications.

However, recent developments demonstrate that the boundary between ancestry-related and health-related genetic testing is more porous than previously suggested [8], and it is being transgressed in a variety of ways. When genetic testing company 23andMe suspended its 'health reports' in 2013, following a warning from the US Food and Drug Administration (FDA), it continued to provide customers with ancestry information and their raw genetic data $[9,10]$. With these ancestry testing data, consumers could still obtain an assessment of their health risks using independent online 'interpretation-only' services for as little as $\$ 5$ [11]. More explicit links between genetic ancestry and health are evident in 23andMe's relaunched Health + Ancestry Service, which was approved by the FDA as a 'medical device' and provides both ancestry and health information. This service directly connects ancestry to health in its 'wellness' reports for traits like lactose intolerance and in its 'carrier status' reports for medical conditions like cystic fibrosis and sickle cell anemia, as both link risk estimates to named racial/ ethnic groups. At least one other leading genetic testing company (Ancestry.com) is in discussions with the FDA about expanding its service to include similar carrier status reports [12]. Furthermore, as we show in this article, even when genetic ancestry tests report only an individual's ancestry, consumers can become aware of possible health risks tied to their genetic ancestry via media coverage of scientific studies and online discussion groups.

This blurring of the line between genetic ancestry and health accentuates the gaps that currently exist in policy and in the available guidance for consumers because little attention has been given to the health implications of genetic ancestry testing. However, as others have noted, it may become common for consumers to share their ancestry test results or ancestry-related estimates of disease risk with their physicians, expecting such information to inform their healthcare decisions and improve their quality of care $[13,14]$. It is therefore crucial that we bridge these gaps to ensure that genetic testing information is used appropriately in health-related decisions and clinical care. This is especially important because most physicians lack the expertise needed to interpret and contextualize the results of genetic tests: only $29 \%$ of US clinicians surveyed rate their knowledge of genetics as excellent, very good, or good [15], and less than a third of the physicians surveyed in five European countries were confident or very confident in their ability to carry out basic medical genetic tasks [16]. Given these findings, there is a real risk that consumers or their physicians could make problematic and potentially irreversible healthcare decisions based on inaccurate, misleading, or misinterpreted genetic testing results. Genetic ancestry information has been misinterpreted or over-interpreted in the past [17-19], and it has been used in ways that reach far beyond the intended or anticipated scientific applications - for example, in controversial attempts to use genetic ancestry tests to support Native American tribal membership claims [20] and to infer nationality in asylum cases [21].

Thus, it is critical that we recognize and address the increasingly porous boundary between genetic tests for ancestry and health because (1) genetic ancestry tests can provide information that has consequences for health decision-making, (2) test-takers may have unrealistic expectations about the scientific and medical certainties offered by the tests they have purchased, (3) many physicians are not prepared to interpret and apply the genetic test results that their patients may bring into the clinic, and (4) better guidance for consumers is needed to ensure that health-related information from genetic ancestry tests is interpreted and applied in valid ways. This is especially important because, as we show in the next section, scientific and biomedical studies have been drawing ever more connections between ancestry and health, and there is evidence that these connections are beginning to affect consumer interpretations of their genetic ancestry test results.

\section{Mounting evidence of connections between genetic ancestry and disease risk}

Genetic ancestry plays an important role in contemporary biomedical science. Medical genetic studies, for instance, 
commonly use ancestry inferences derived from autosomal markers (typically single nucleotide polymorphisms or "ancestry informative markers") to control for population stratification, a practice that underpins the now routine reporting of population-specific or ancestry-specific estimates of disease risks and drug response in genetic epidemiology $[13,22]$. Far less attention has been given to the fact that studies using uniparental genetic markers have uncovered connections between ancestry and health. These tests have been a mainstay of the direct-to-consumer ancestry-testing marketplace, but have been widely considered to have little biomedical value [13].

Over the last decade, hundreds of biomedical studies have been published that suggest that certain mitochondrial DNA (mtDNA) and Y chromosome variants (and the haplogroups defined by those variants) are associated with an increased risk of disease and other health complications [23]. These variants and haplogroups have been linked to a diverse array of common diseases and medical conditions, including coronary artery disease, myocardial infarction, ischaemic stroke, heart transplant complications, Leber hereditary optic neuropathy, advanced age-related macular degeneration, hearing loss, osteoarthritis, osteoporosis, multiple sclerosis, Alzheimer's and Parkinson's disease, complications from type 2 diabetes (especially retinopathy, neuropathy, nephropathy, and renal failure), several types of cancer (breast, thyroid, pancreatic, esophageal, colorectal, prostate, renal, and lung cancer), and the rate of AIDS progression in HIV patients (an additional table shows examples of associations discussed in the biomedical literature [see Additional file 1]). The exact causes of these associations are not always clear, but it is thought that the associated genetic variants alter the expression of key gene pathways [24] or, in the case of mtDNA, contribute to the development and progression of disease by affecting energy metabolism and important cellular processes, including ATP synthesis, reactive oxygen species (ROS) production, oxygen consumption efficiency, calcium signaling, and apoptosis [25-27].

It is important to note that the quality of studies reporting associations between mtDNA/Y chromosome haplogroups and disease susceptibility is quite variable. Some haplogroup-disease associations are supported by multiple independent studies with rigorous statistical designs, but many others are not. The biomedical literature includes a large number of studies that suffer from small sample sizes, inappropriate controls for population stratification, and/or problematic statistics (for example, $P$-values that have not been corrected for multiple comparisons), so some of the reported haplogroup-disease associations are almost certainly false positives. Other researchers have also drawn attention to these problems $[23,28]$. However, regardless of the quality of these studies, many publications present possible associations between mtDNA/Y haplogroups and disease risk, and consumers are becoming aware of these reports as they garner media attention and enter public discourse.

In 2012, for example, a study published in The Lancet found that British men belonging to $\mathrm{Y}$ chromosome haplogroup I have a 50\% higher age-adjusted risk of coronary artery disease (CAD) than other British men, with haplogroup I being the most significant predictor of CAD after HDL cholesterol and lipid-lowering treatment [24]. This finding was widely reported by the media in the UK, US, and Australia, under headlines about heart disease risk being inherited along paternal lines (Tables 1 and 2). This coverage demonstrated the potential for raised consumer expectations about the value of genetic ancestry information to health, and the future possibilities of acting on that information. One article, for example, suggested that: "when a screening test is developed to find those $\mathrm{Y}$ chromosome gene clusters and researchers have a better understanding of how they act, it may be possible to protect some [unlucky men] from having heart attacks." [29] The UK National Health Service added an extensive discussion of ancestral haplogroups and CAD to their patient information website, NHS Choices, after the study was published - albeit to argue that this information was not of immediate use for tackling CAD in the UK because, among other reasons, "men are unlikely to know their specific haplogroup, so are unlikely to know whether they may be at increased risk of CAD" [30]. Ancestry testing consumers discussed this Lancet study (along with other reports of haplogroup-associated disease susceptibilities) in online forums (in threads entitled, for example, "Medical conditions associated with Y-chromosome haplogroups" and "Do not read if you are a hypochondriac..."), and a Principal Scientist at 23andMe blogged about the study, expressing skepticism about the study's conclusions and offering an alternative analysis using his company's data (Table 3). Thus, by following the circulation of this study in public domains, we can see variation in how the study was understood, disagreement over the robustness of its conclusions, and a lack of clarity about the significance of genetic ancestry markers like haplogroups to health.

While similar observations can be made about studies of autosomal or genome-wide markers and disease risk, we have focused here on uniparental genetic markers because mtDNA and Y chromosome tests are the two types of genetic ancestry tests that companies, policymakers, regulators, and professional scientific associations have invariably treated as less relevant to health. However, as we have shown, there is public interest in the extensive biomedical literature investigating the associations between mtDNA/Y chromosome haplogroups 


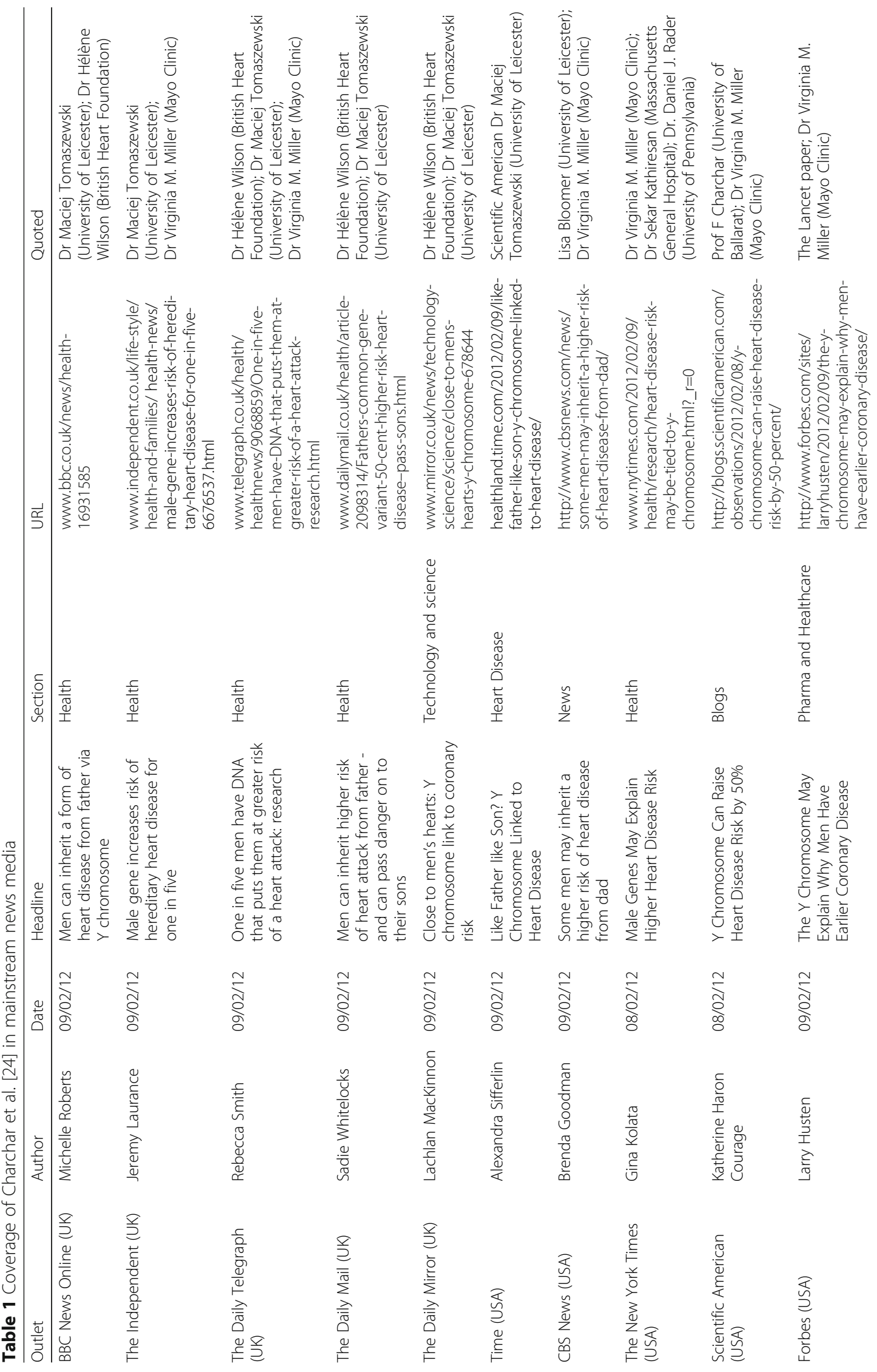




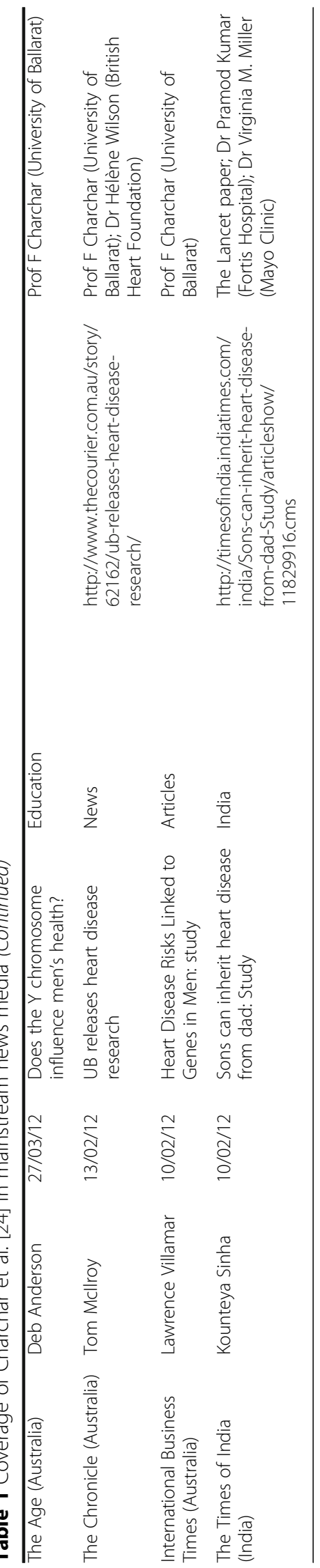




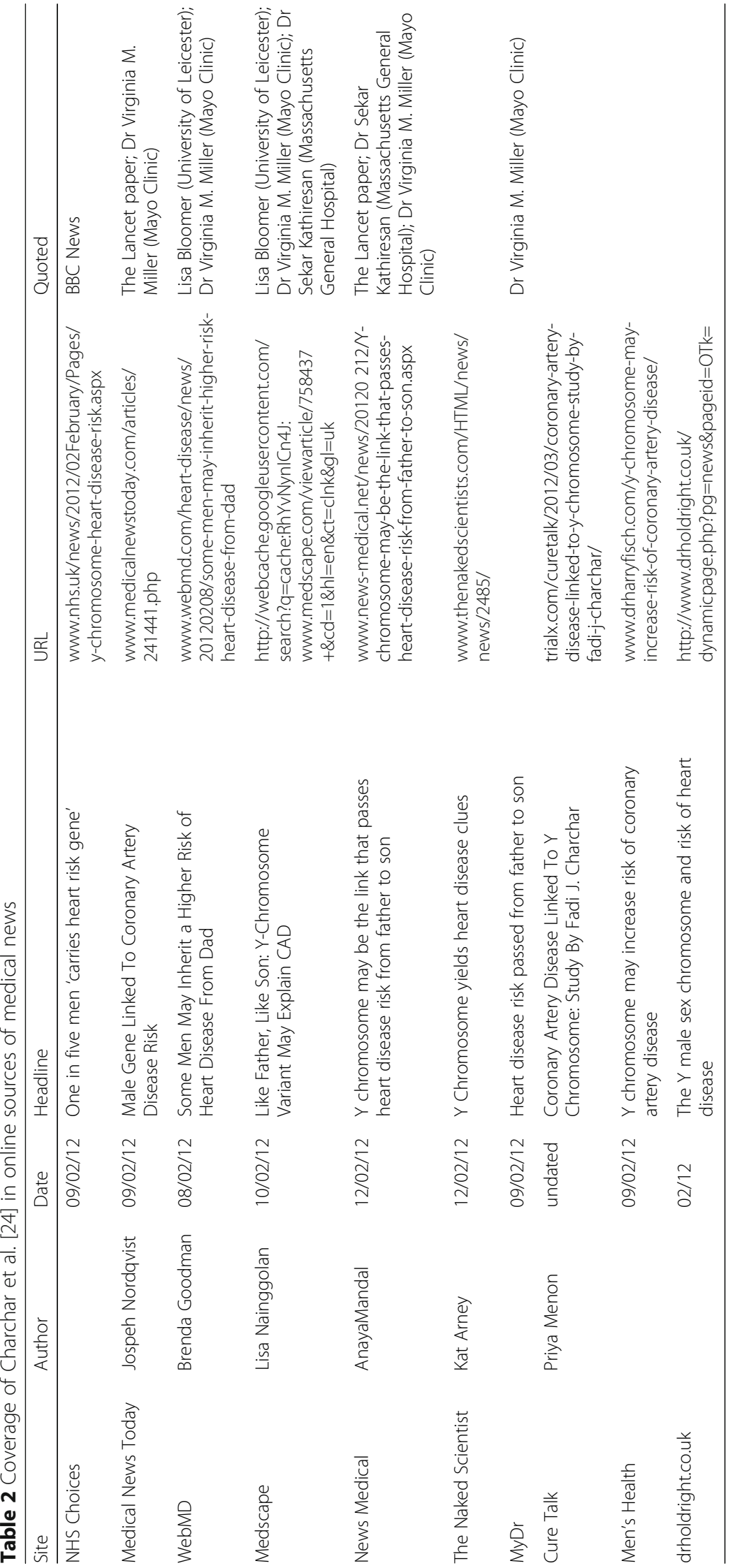




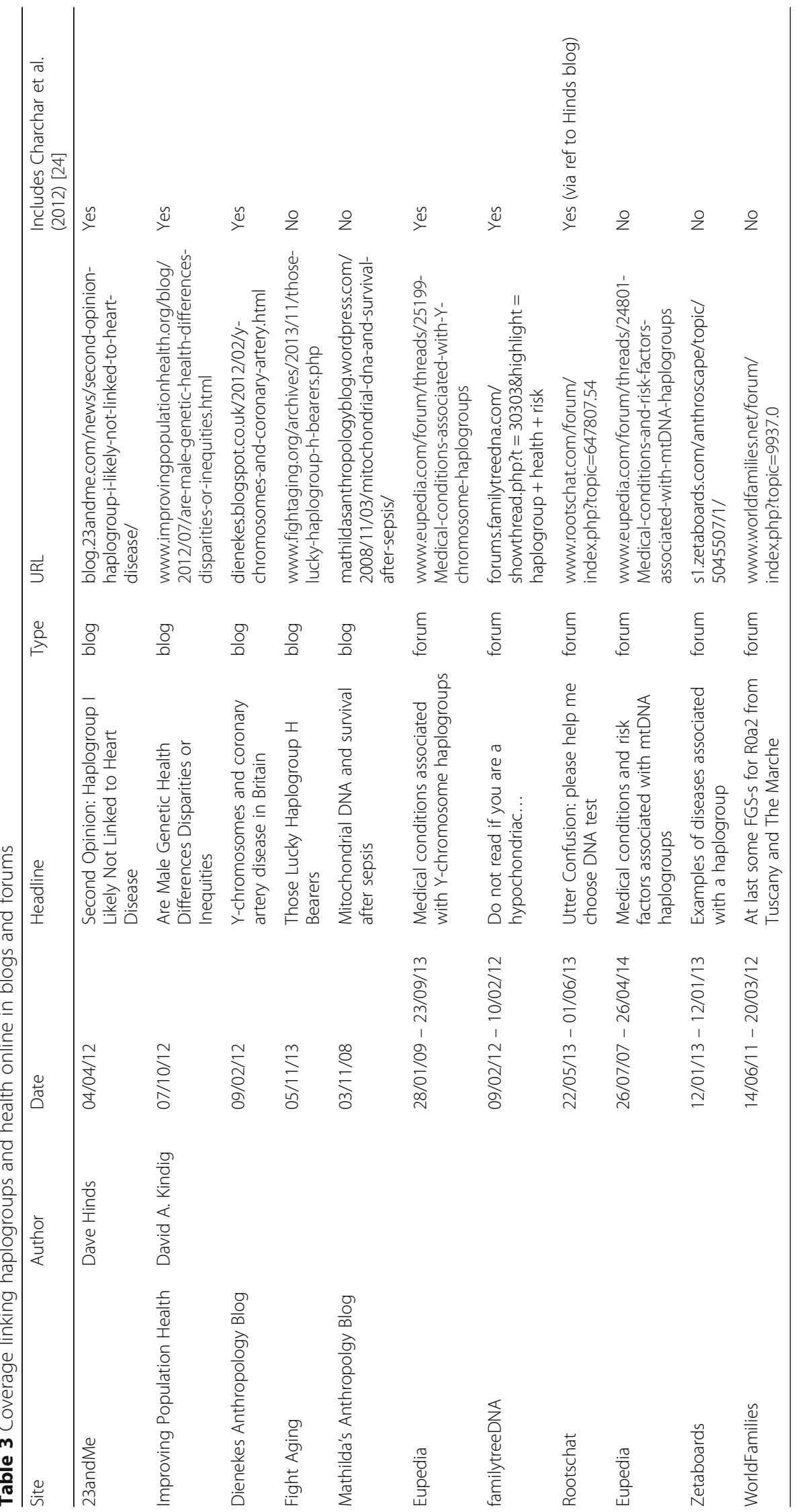


and disease, and some consumers of genetic ancestry tests are already trying to understand how their ancestry is relevant to their health and disease prognosis in light of these research findings. Other consumers are also likely to encounter the results of these biomedical studies in the popular press, in online forums, in literature from their healthcare provider, or in well-known medical journals, and they too may grapple with the possible health implications of their genetic ancestry test results. Therefore, even when genetic ancestry tests report only an individual's ancestral lineage or uniparental haplogroup, consumers can become aware of the possible relationship with genetic health risks because scientists have linked haplogroup ancestry to health outcomes.

\section{Conclusions}

Like the repurposing of genome-wide ancestry test data to assess health risks, and like the use of autosomal genetic markers in genetic epidemiology to identify population-specific disease risks and drug response, the reported associations between uniparentally-inherited haplogroups and various diseases represent another blurring of the line between genetic information on health and ancestry. Developments in scientific knowledge and commercial practice appear to be outpacing the current oversight and governance regimes that largely treat genetic tests for ancestry and health as separate and distinct.

We therefore suggest that it is time for the various stakeholders in genetic ancestry testing to reconsider what they communicate about the health implications of ancestry information, both to the public and to each other. This will require considering some difficult questions. For example, can and should consumer genetic testing companies take responsibility for the ways in which test-takers connect ancestry test results with other publicly available information? Can and should genetic testing companies, alongside scientific associations and consumer advocates, provide guidance to consumers about the accuracy and reliability of the various postulated associations between haplogroups (or other genetic markers) and health risks? How can those who communicate about the relevant science (scientific researchers, journal editors, science journalists, genetic testing companies, consumer advocates, policy advisors, etc.) achieve maximum clarity regarding the potential health implications of genetic ancestry (or the lack of them), and make effective use of the published critiques of the putative links between uniparental haplogroups and health risks $[23,28]$ ? Should there be any changes to policies governing the regulation or oversight of consumer genetic testing, or additions to guidelines being developed by professional associations like the American Society of Human Genetics (ASHG)?

To help address the issues raised here, we make five recommendations for stakeholders in consumer genetic testing to consider:

(1) The ASHG or another respected professional genetics society should organize a roundtable to bring the various stakeholders together to produce authoritative guidance that will inform and benefit consumers, and help create a set of standards for the industry to follow. This guidance should make clear what we can and cannot know from genetic ancestry testing, and provide guidance regarding the accuracy and reliability of associations between genetic markers and health risks. Industry representatives, consumer advocates, policy and legal advisors, biomedical researchers, social scientists, and regulators should all be involved in drafting this guidance.

(2) Consumer advocates, scientific organizations, companies, science journalists, and government agencies should play a role in making this information available to consumers.

(3) Genetic ancestry testing companies should report only associations between genetic markers and diseases/medical conditions that have been scientifically validated. They should also provide information about the limitations of their tests (as 23andMe now does as part of their 'carrier status' and 'wellness' reports; [31]) and include information about how to interpret the connections between ancestry and health among their FAQs.

(4) Medical schools and continuing medical education (CME) programs should discuss the potential health implications of genetic ancestry information so that physicians can help their patients to interpret and contextualize their genetic testing results.

(5) Policy-makers and government agencies may wish to reconsider current oversight regimes for direct-to-consumer genetic testing in light of the increasingly porous boundaries between tests for health and ancestry.

Our recommendations are aimed at encouraging novel and timely interventions into ongoing debates about direct-to-consumer genetic tests, especially since the US FDA, European Commission, ASHG, and high-profile ancestry testing companies are all considering scientific, ethical, and regulatory issues regarding health-related genetic testing. Now is the time to start bridging the gap in our current approaches to health and ancestry genetic testing. 


\section{Additional file}

Additional file 1: Examples of associations between mitochondrial DNA (mtDNA) or $Y$ chromosome variants and diseases/medical traits discussed in the biomedical literature. Description of data: an extensive list of published associations between mitochondrial DNA (mtDNA) or $Y$ chromosome variants and diseases/medical traits. Organised by disease/medical traits, and including: mtDNA or Y Chromosome Variant; Proposed Association (or Lack of Association); Study Location; and Reference. (DOCX 58 kb)

\section{Abbreviations}

ASHG: American Society of Human Genetics; CAD: Coronary artery disease; CME: Continuing medical education; FDA: Food and Drug Administration; mtDNA: Mitochondrial DNA; PGS: Personal genome service; ROS: Reactive oxygen species

\section{Acknowledgments}

None.

\section{Availability of data and materials}

The datasets supporting the conclusions of this article are included within the article.

\section{Authors' contributions}

All authors contributed substantively to the argument developed in this paper and to the drafting and editing of the manuscript. AS initiated the collaboration, and collated and reviewed evidence from print and online media sources. RT advised on ethical and legal frameworks. AS and DB collated evidence of scientific work in the field, and DB reviewed and evaluated these. All authors read and approved the final manuscript.

\section{Competing interests}

The authors declare that they have no competing interests.

\section{Consent for publication}

Not applicable.

\section{Ethics approval and consent to participate}

Not applicable.

\section{Author details}

${ }^{1}$ Department of Sociology, Bath Spa University, Newton Park, Bath BA2 9BN UK. ${ }^{2}$ Department of Anthropology, University of Texas at Austin, 2201 Speedway, Stop C3200, Austin, TX 78712-1723, USA. ${ }^{3}$ Department of Sociology, Lancaster University, Bowland North, Bailrigg LA1 4YN, UK.

Received: 30 March 2016 Accepted: 21 December 2016

Published online: 09 January 2017

References

1. Kaiser J. Who has your DNA —or wants it. Science. 2015;349:1475.

2. Wells S. Paper presented at the International Conference for Genetic Genealogy. 2014

3. Wagner J, et al. Tilting at windmills no longer: a data-driven discussion of DTC DNA ancestry tests. Genet Med. 2012;14:586-93.

4. Kalokairinou $L$, et al. Changes on the horizon for consumer genomics in the EU. Science. 2014;346:296-8. doi:10.1126/science.1256396.

5. Hudson K et al. ASHG Statement on Direct-to-Consumer (DTC) Genetic Testing in the United States. Am Soc Hum Genet. 2007;81:635-7.

6. The American Society of Human Genetics. ASHG Ancestry Testing Statement and Recommendations: guidelines for Understanding the issues and implications involved. 2008. Available: www.ashg.org/pages/policy_ statements shtml.

7. National Human Genome Research Institute. Regulation of genetic tests. 2014. Available: www.genome.gov/10002335.

8. Lee $\mathrm{S}$, et al. The illusive gold standard in genetic ancestry testing. Science. 2009;325:38-9.

9. U.S. Food and Drug Administration. Warning letter: 23andMe, Inc. 2013. Available: www.fda.gov/iceci/enforcementactions/warningletters/2013/ ucm376296.htm
10. Seon-Hee Y, Chung Y-J. Reflections on the US FDA's Warning on Direct-toConsumer Genetic Testing. Genomics Inform. 2014;12(4):151-5. doi:10.5808/ GI.2014.12.4.151.

11. Regalado A. How a wiki is keeping direct-to-consumer genetics alive. MIT technology review. 2014. Available: www.technologyreview.com/ featuredstory/531461.

12. Duhaime-Ross A. Ancestry.com is talking to the FDA about using DNA to estimate people's risk of disease. The Verge. 2015. Available: http://www. theverge.com/2015/10/12/9487685/ancestry-com-dna-test-kit-disease-risk-fda.

13. Royal C, et al. Inferring genetic ancestry: opportunities, challenges, and implications. Am J Hum Genet. 2010:86:661-73.

14. Cherkas $L$, et al. A survey of UK public interest in internet-based personal genome testing. PLoS One. 2010;5(10):e13473.

15. Bonham $V$, et al. Physicians' knowledge, beliefs, and use of race and human genetic variation: new measures and insights. BMC Health Serv Res. 2014;14:456

16. Nippert I, et al. Confidence of primary care physicians in their ability to carry out basic medical genetic tasks - a European survey in five countries-Part 1. J Community Genet. 2011;2(1):1-11.

17. Richards M. Beware the gene genies. The Guardian. 2003. Available: http:// www.theguardian.com/education/2003/feb/21/highereducation.uk.

18. Bolnick D, et al. Genetics. The science and business of genetic ancestry testing. Science. 2007:318(5849):399-400.

19. Sense about Science. Sense about genetic ancestry testing. 2013. Available: http://archive.senseaboutscience.org/pages/genetic-ancestry-testing.html.

20. Tallbear K. DNA, blood, and racializing the tribe. Wicazo Sa Review. 2003; 18(1):81-107.

21. Tutton R, Hauskeller C, Sturdy S. Suspect technologies: forensic testing of asylum seekers at the UK border. Ethnic Racial Stud. 2014;37:738-52.

22. $\mathrm{Yu} \mathrm{J-H}$, et al. What are our AlMs? Interdisciplinary perspectives on the use of ancestry estimation in disease research. AJOB Prim Res. 2012;3:87-97.

23. Salas A, Elson JL. Mitochondrial DNA as a risk factor for false positives in case-control association studies. J Genet Genomics. 2015;42:169-72.

24. Charchar $F$, et al. Inheritance of coronary artery disease in men: an analysis of the role of the $Y$ chromosome. Lancet. 2012;379:915-22.

25. Santoro A, et al. Evidence for sub-haplogroup $\mathrm{H} 5$ of mitochondrial DNA as a risk factor for late onset Alzheimer's disease. PLoS One. 2010;5:e12037. doi:10.1371/journal.pone.0012037.

26. Ebner $\mathrm{S}$, et al. Mitochondrial haplogroups, control region polymorphisms and malignant melanoma: a study in Middle European Caucasians. PLoS One. 2011;6:e27192. doi:10.1371/journal.pone.0027192.

27. Palacín M, et al. Mitochondrial DNA and TFAM gene variation in early-onset myocardial infarction: evidence for an association to haplogroup $\mathrm{H}$. Mitochondrion. 2011;11:176-81.

28. Raule $\mathrm{N}$, et al. Association studies on human mitochondrial DNA: methodological aspects and results in the most common age-related diseases. Mitochondrion. 2007:7:29e38.

29. Kolata G. Male genes may explain higher heart disease risk. The New York Times. 2012. Available: http://www.nytimes.com/2012/02/09/health/ research/heart-disease-risk-may-be-tied-to-y-chromosome.html.

30. Choices NHS. One in five men 'carries heart risk gene'. 2012. Available: www. nhs.uk/news/2012/02February/Pages/y-chromosome-heart-disease-risk.aspx.

31. 23andMe. Learn how your DNA may affect your health. 2016. Available: www 23andme.com/service/. 